\title{
Postrelapse Survival in Osteosarcoma of the Extremities: Prognostic Factors for Long-Term Survival
}

\author{
By Stefano Ferrari, Antonio Briccoli, Mario Mercuri, Franco Bertoni, Piero Picci, Amelia Tienghi, Adalberto Brach Del Prever,
} Franca Fagioli, Alessandro Comandone, and Gaetano Bacci

\begin{abstract}
Purpose: To identify factors that influence postrelapse survival (PRS) in patients with nonmetastatic osteosarcoma of the extremity

Patients and Methods: One hundred sixty-two patients with recurrent osteosarcoma of the extremity were retrospectively reviewed. The first-line treatment included surgery of the primary lesion and chemotherapy with methotrexate, doxorubicin, cisplatin, and ifosfamide.

Results: The projected 5-year PRS rate was 28\%. Patients who had complete surgery of recurrence had a 5-year PRS of $39 \%$, whereas for those who did not have complete surgery, PRS was $0 \%$ at 3 years $(P<.0001)$. In the latter group, PRS was not influenced by site of recurrence and relapse-free interval (RFI), although it was influenced $(P=$ .006) by the use of second-line chemotherapy (PRS, 53\% at 12 months for patients who received chemotherapy $v 12 \%$
\end{abstract}

$\mathrm{H}$ IGH-GRADE osteosarcoma is a malignant bony tumor with a peak of incidence in the second decade of life. When treated by surgery alone, usually amputation, the cure rate was approximately $10 \%$, but the use of chemotherapy has significantly improved the clinical outcome. ${ }^{1}$

Recurrent disease still occurs in approximately $30 \%$ to $40 \%$ of patients with nonmetastatic osteosarcoma of the extremity despite a complete surgical removal of the tumor and intensive chemotherapeutic treatment. $^{2-6}$ The postrelapse survival (PRS) in this subset of chemotherapy-resistant patients is usually low. An aggressive and even repeated pulmonary metastasectomy is at present an accepted strategy of treatment in patients with metastases confined to the lung, ${ }^{7-16}$ whereas the value of secondline chemotherapy treatment ${ }^{9,10,12-16}$ and the best strategy of treatment for those patients with recurrence other than that of the lung ${ }^{11,14,15,17}$ are not well defined.

This study is a retrospective analysis of patients who relapsed from high-grade osteosarcoma of the extremity. The aim of the study was to identify factors influencing the PRS.

\section{PATIENTS AND METHODS}

\section{Patients}

Patients with a diagnosis of nonmetastatic osteosarcoma of the extremity who entered four sequential studies carried out at our institute between

From the Department of Musculoskeletal Oncology at Istituto Ortopedico Rizzoli, Bologna; Medical Oncology Ravenna, Ravenna; Pediatric Division, University Hospital; and Medical Oncology, Gradenigo Hospital, Torino, Italy. Submitted March 26, 2002; accepted October 30, 2002.

Address reprint requests to Stefano Ferrari, MD, Sezione di Chemioterapia, Istituto Ortopedico Rizzoli, Via Pupilli 1, 40136, Bologna, Italy; email: stefano.ferrari@ior.it.

(C) 2003 by American Society of Clinical Oncology.

0732-183X/03/2104-710/\$20.00 for those who did not). In patients who had complete surgery, PRS was influenced by site of relapse (5-year PRS, lung 44\%, other 19\%; $P<.06)$, RFI (5-year PRS at $\leq 24$ months, 20\%; at $>24$ months, 60\%; $P<.0001$ ), and number of lung metastases (5-year PRS, two or fewer nodules, 59\%; more than two nodules, $14 \% ; P<.0001$ ) but not by the use of a second-line chemotherapy treatment.

Conclusion: RFI, site of metastases, and number of pulmonary nodules are the main prognostic factors for PRS in osteosarcoma. Complete surgery of recurrence is pivotal in the strategy of treatment. Patients with unresectable recurrence benefit from second-line chemotherapy, whereas our data do not support a generalized use of chemotherapy after complete surgery of first recurrence.

J Clin Oncol 21:710-715. () 2003 by American Society of Clinical Oncology.

October 1986 and June 1995 were identified using the database of the tumor center and chemotherapy department of the authors' institutions.

Between October 1986 and June 1995, four protocols for the treatment of nonmetastatic osteosarcoma of the extremity were sequentially activated at the Rizzoli Institute: IOR/OS-2, IOR/OS-3, IOR/OS-3bis, and IOR/OS-4. Characteristics and results have been previously reported in detail. ${ }^{3,18-20}$

The criteria of eligibility for IOR/OS-2, IOR/OS-3, IOR/OS-3bis, and IOR/OS-4 were the following: biopsy-proven, classic, high-grade osteosarcoma of the extremity; age younger than 40 years; absence of metastases confirmed by bone scintigraphy and by computed tomography of the lungs; an interval of less than 1 month between biopsy and start of chemotherapy; normal renal and hepatic function; and no prior chemotherapy or surgical treatment for the bone lesion.

The strategy of treatment was based on primary chemotherapy treatment, resection of the primary tumor, and postoperative chemotherapy treatment (neoadjuvant treatment). In the first three protocols (IOR/OS-2, IOR/OS-3, and IOR/OS-3bis), patients received primary chemotherapy with high-dose methotrexate (MTX), cisplatin (CDP), and doxorubicin (ADM). Postoperatively, patients with a good histologic response received the same drugs preoperatively used, whereas in those with a poor histologic response, ifosfamide (IFO) was added to the three drugs preoperatively used. In the fourth protocol, all the patients received IFO from the preoperative phase added to MTX, CDP, and ADM.

After chemotherapy, patients were checked by radiographs of the chest and the treated limb and observed in the outpatient clinic every 2 months for 2 years, every 3 months in the third year, and then every 6 months thereafter. In the last protocol (IOR/OS-4), most of the patients were checked with computed tomography scans of the lung performed every 3 months in the first 3 years, every 4 months in the fourth and fifth years, and then every 6 months thereafter.

Because the pattern of relapse of osteosarcoma is heterogeneous, the strategy of treatment of first relapse was individualized. Nevertheless, the cornerstone of treatment was the surgical removal of the metastases whenever possible. A second round of chemotherapy treatment was usually suggested only when it was not possible to achieve complete surgical remission or when the metastatic pattern suggested a particularly aggressive behavior (multiple metastases or short disease-free interval). Since 1991, patients have been receiving four cycles of high-dose IFO $\left(15 \mathrm{~g} / \mathrm{m}^{2}\right.$ over 5 days continuous infusion). In previous years, patients usually received different treatments based on drug combinations not used in first-line 
chemotherapy, such as bleomycin, $15 \mathrm{mg} / \mathrm{m}^{2} / \mathrm{d}$ for 2 days, cyclophosphamide, $600 \mathrm{mg} / \mathrm{m}^{2} / \mathrm{d}$ for 2 days, and dactinomycin, $600 \mu \mathrm{g} / \mathrm{m}^{2} / \mathrm{d}$ for 2 days; $\mathrm{CDP}, 120 \mathrm{mg} / \mathrm{m}^{2}, 72$ hours continuous infusion, and etoposide, $120 \mathrm{mg} / \mathrm{m}^{2} / \mathrm{d}$ for 3 days; cyclophosphamide, $300 \mathrm{mg} / \mathrm{m}^{2}$ every 12 hours for six doses, and etoposide, $200 \mathrm{mg} / \mathrm{m}^{2} / \mathrm{d}$ for 3 days; or standard-dose IFO, $2 \mathrm{~g} / \mathrm{m}^{2} / \mathrm{d}$ for 5 days.

Four hundred thirty-five patients entered the IOR/OS-2, IOR/OS-3, IOR/OS-3bis, and IOR/OS-4 protocols. With a follow-up ranging between 6 and 12 years (median, 8.5 years), the 10-year disease-free survival was $57 \%$ (95\% confidence interval [CI], 52\% to $62 \%$ ). One hundred seventy-five patients (40\%) had recurrence.

\section{Statistical Methods}

The aim of this study was to identify factors that influence the PRS in patients with initial diagnosis of nonmetastatic classic high-grade osteosarcoma of the extremity. For this analysis, patients were eligible when it was possible to collect information on date and type of first relapse (local recurrence and/or distant metastases), treatment after relapse, and date and cause of death. Information was collected from database files, pathology and radiology reports, and general medical records. Complete surgical remission was defined as removal of all evident metastatic disease, with no tumor tissue at the resection margins on histologic examination.

PRS was calculated from the date of recurrence until death or last follow-up. The following parameters were investigated: relapse-free interval (RFI; time between surgical resection of the primary tumor and date of onset of relapse), pattern of first relapse (lung metastases, bone metastases, local recurrence, and other sites of metastases), site (monolateral or bilateral) of lung metastases, number of resected pulmonary nodules, surgical complete remission, and second-line chemotherapy treatment.

The Statview 4.5 statistical package (Abacus Concepts, Berkeley, CA) was used for the statistical analysis. Differences between the groups were compared by means of the $\chi^{2}$ test and $t$ test. Survival curves were calculated according to the Kaplan-Meier method and compared by means of the log-rank test.

\section{RESULTS}

Of the 175 patients who had recurrence, 59 (34\%) were treated according to IOR/OS-2 protocol, 45 (26\%) were in IOR/OS-3, $24(14 \%)$ were in IOR/OS-3 bis, and $47(27 \%)$ were in IOR/OS-4 protocols. One hundred sixty-two patients $(92.5 \%)$ were eligible for the analysis; 13 (six in IOR/OS-2, three in IOR/OS-3, one in IOR/OS-3bis, and three in IOR/OS-4) were excluded because after relapse they preferred to move to other centers, and no information about treatment received was available. Patient characteristics and pattern of first relapse are reported in Table 1. There was a prevalence of lung location; overall, 136 patients $(84 \%)$ had pulmonary metastases, confined only to the lung in 125 patients. Regarding relapse-free interval, 85 patients $(52.5 \%)$ relapsed in the first 2 years of follow-up (20 in the first year), 42 (26\%) relapsed in the third year, $19(12 \%)$ relapsed in the fourth year, and seven relapsed in the fifth year. Afterwards, recurrence was recorded only in nine patients (5.5\%); the latest recurrence occurred after 11 years and 5 months of follow-up.

Surgery of metastatic lesions was performed in 125 patients (77\%). In 11 of them, the surgical procedure was oncologically inadequate, with residual of the metastases. Overall, 114 patients (70\%) achieved complete surgical remission. Of the 125 patients with metastases confined to the lung, 93 (74.5\%) were free of disease after complete surgical treatment, as opposed to 21 (57\%) of the 37 patients with other than lung metastases $(P=$ .04). Among the 93 patients in complete surgical remission, the RFI was $\leq 24$ months in 44 patients $(47 \%)$ and longer than 24 months in 49 patients (53\%); 64 patients $(69 \%)$ had one to two
Table 1. Patient Characteristics and Pattern of First Relapse

\begin{tabular}{|c|c|c|}
\hline Characteristic & $\begin{array}{l}\text { No. of } \\
\text { Patients }\end{array}$ & $\%$ \\
\hline \multicolumn{3}{|l|}{ Age at first relapse, years } \\
\hline Median & \multicolumn{2}{|c|}{17.5} \\
\hline Range & \multicolumn{2}{|c|}{5 to 47} \\
\hline \multicolumn{3}{|l|}{ Relapse-free interval, months } \\
\hline Median & \multicolumn{2}{|c|}{23} \\
\hline Range & \multicolumn{2}{|c|}{2 to 137} \\
\hline \multicolumn{3}{|l|}{ Site } \\
\hline Lung only & 125 & 77 \\
\hline Monolateral & 80 & 64 \\
\hline Bilateral & 45 & 36 \\
\hline Other site \pm lung & 37 & 23 \\
\hline Bone \pm local recurrence & 23 & 62 \\
\hline Bone and lung & 8 & 22 \\
\hline Local recurrence and lung & 3 & 8 \\
\hline Lymph nodes & 2 & 5 \\
\hline Heart & 1 & 3 \\
\hline
\end{tabular}

pulmonary nodules, and $29(31 \%)$ had three or more nodules. None of the patients with bone and lung metastases achieved a disease-free status, whereas a complete surgery was performed in 17 of the patients (74\%) with bone metastases or local recurrence, in two of the three patients with local recurrence and lung metastases, and in two patients with lymph node metastases. Patients who had complete surgical remission had a longer RFI (mean \pm SD, $28 \pm 18$ months) than those who did not (mean $\pm \mathrm{SD}, 23 \pm 13.5$ months), but the difference was not statistically significant $(P=.85)$.

In the 93 patients with lung metastases only and who received oncologically adequate surgical treatment, the surgical procedures were monolateral wedge resection in 54 patients (58\%), bilateral wedge resection in 22 patients (24\%), lobectomy with or without wedge resection in 15 patients (16\%), and pneumonectomy in two patients. The mean number of resected metastatic nodules was 2.7 (range, one to 12 nodules). Of the 23 patients with bone metastases and or local recurrence, 17 (74\%) had complete surgery after amputation (10 patients) or resection with prosthetic reconstruction (seven patients), whereas six patients could not achieve a complete surgical remission. Contemporary resection of local recurrence and lung metastases (monolateral wedge resection) was performed in two patients. Two patients with lymph node metastases underwent resection.

Second-line chemotherapy treatment was given in 56 of the patients (35\%), high-dose IFO was given to 31 patients (55\%), and other treatments were given to 25 patients (45\%). In 36 patients, chemotherapy followed surgical complete remission, and in six patients, it preceded surgical complete remission; in 14 patients, a surgical treatment was not feasible despite the chemotherapy treatment. In patients who achieved surgical complete remission, second-line chemotherapy was given when the pattern of recurrence suggested a more aggressive behavior of the tumor, such as in patients with other than lung locations and, in case of lung location of metastases, in patents with three or more nodules or a short RFI (Table 2). In patients in surgical complete remission and with lung metastases, two patients with lung metastases and local recurrence received chemotherapy, whereas among patients with only lung metastases, 29 patients 
Table 2. Pattern of Relapse and Use of Second-Line Chemotherapy Treatment

in 114 Patients in Surgical Complete Remission

\begin{tabular}{|c|c|c|c|c|c|}
\hline \multirow[b]{2}{*}{ Variable } & \multicolumn{2}{|c|}{$\begin{array}{l}\text { Surgery + Chemotherapy } \\
\qquad(\mathrm{n}=42)\end{array}$} & \multicolumn{2}{|c|}{$\begin{array}{l}\text { Surgery } \\
(\mathrm{n}=72)\end{array}$} & \multirow[b]{2}{*}{$P$} \\
\hline & No. of Patients & $\%$ & No. of Patients & $\%$ & \\
\hline Site & & & & & .01 \\
\hline Lung only & 29 & 31 & 64 & 69 & \\
\hline Other sites \pm lung & 13 & 62 & 8 & 38 & \\
\hline Relapse-free interval & & & & & .7 \\
\hline$\leq 24$ months & 22 & 39 & 34 & 61 & \\
\hline$>24$ months & 20 & 34 & 38 & 66 & \\
\hline Laterality* & & & & & .3 \\
\hline Monolateral & 21 & 29 & 51 & 71 & \\
\hline Bilateral & 10 & 43 & 13 & 57 & \\
\hline Lung metastases* & & & & & $<.0001$ \\
\hline$\leq 2$ nodules & 14 & 21 & 52 & 79 & \\
\hline$>2$ nodules & 17 & 59 & 12 & 41 & \\
\hline
\end{tabular}

*Of 95 patients, 93 had only lung metastases, and two had monolateral lung metastases and local recurrence.

(31\%) received chemotherapy and $64(69 \%)$ did not. All 48 patients (30\%) who did not have complete surgical remission died of disease, with a median PRS ranging between 1 and 26 months (median, 6 months).

Of the 114 patients surgically free of disease, 25 (22\%) were continuously free of disease, with a median PRS of 60 months (range, 20 to 112 months). Eighty-nine patients (78\%) relapsed with a median second RFI of 8 months (range, 1 to 54 months). Of those patients, $20(22.5 \%)$ were alive after repeated surgical procedures for the metastatic disease, with a median time after the last metastatic event ranging from 2 to 106 months (median, 45 months). Overall, with a median first PRS time of 65 months (range, 12 to 131 months), 45 of the patients (39.5\%) in complete surgical remission after first recurrence were alive, 40 were alive without evidence of disease, and five were alive with disease.

The actuarial probability of PRS on the whole population was $33 \%(95 \% \mathrm{CI}, 25 \%$ to $40 \%)$ at 3 years and $28 \%$ (95\% CI, $21 \%$ to $35 \%)$ at 5 years. Those patients who had complete surgical remission had a probability of PRS at 5 years of $39 \%$ (95\% CI, $30 \%$ to $49 \%$ ); whereas those who did not had a $2 \%$ (95\% CI, $0 \%$ to $7 \%$ ) 2-year probability of PRS and a $0 \%$ probability at 3 years $(P<.001)$. According to the site of first recurrence, patients who had recurrence confined to the lung had a 5-year PRS of 33\% (95\% CI, $24 \%$ to $42 \%$ ), and those with a recurrence location other than the lung had a PRS of $11 \%(95 \%$ CI, $1 \%$ to $21 \%)$ at 5 years $(P=.013)$. According to the RFI, the 5-year PRS probability was $5 \%(95 \% \mathrm{CI}, 0 \%$ to $14 \%)$ for patients having a first RFI ranging between 1 and 12 months, $15 \%$ (95\% CI, $7 \%$ to 24\%) when the RFI was between 13 and 24 months, 38\% (95\% CI, $23 \%$ to 53\%) when it was between 25 and 36 months, and $59 \%$ (95\% CI, $41 \%$ to $77 \%$ ) for patients having a longer RFI $(P<.001)$.

In patients who failed to achieve complete surgical remission, the PRS probability was not different according to the site of first recurrence and the length of the RFI, whereas it was influenced by the use of chemotherapy (53\% PRS [95\% CI, 28\% to 78\%] at 12 months for patients who received chemotherapy $v 12 \%$ PRS [95\% CI, $1 \%$ to $23 \%$ ] for patients who did not; $P=.006$ ). At 2 years, the PRS probability was 0 in the two groups.
In patients who reached complete surgical remission, the postrelapse 5-year probability of event-free survival (EFS) was $16 \%$ (95\% CI, $8 \%$ to $23 \%$ ). In patients with only lung recurrence, the 5-year EFS was $18 \%$ (95\% CI, 9\% to 27\%), and it was $6 \%(95 \% \mathrm{CI}, 0 \%$ to $18 \%)$ for other locations of recurrence $(P=$ .5). Patients with an RFI longer than 24 months had a 24\% (95\% CI, $12 \%$ to $36 \%$ ) 5 -year EFS, which was significantly better $(P<.0001)$ than that of those with a shorter RFI $(9 \%$ EFS; 95\% CI, $1 \%$ to $17 \%)$. When metastases were localized to the lung, a significantly better 5 -year EFS $(P<.008)$ was found in patients with only one to two pulmonary nodules $(24 \% ; 95 \%$ CI, $12 \%$ to $36 \%$ ) compared with that of patients with a higher number of lung metastases (3\% EFS; $95 \%$ CI, $0 \%$ to $10 \%$ ). Patients with monolateral lung metastases had a better 5-year EFS (20\%; $95 \%$ CI, $9 \%$ to $31 \%$ ) than those with bilateral metastases (8\% EFS; 95\% CI, $0 \%$ to $19 \%$ ). A 5-year EFS of $10 \%$ (95\% CI, $0 \%$ to $20 \%$ ) was found in patients who received a second-line chemotherapy treatment, whereas it was $19 \%$ (95\% CI, $8 \%$ to $29 \%)$ when chemotherapy was not administered $(P=.1)$

In patients who reached complete surgical remission, a different probability of PRS was documented according to several variables (Table 3). Patients with metastases confined to the lung had a better prognosis than patients with metastases in other locations. According to the RFI, the 5-year PRS probability was $8 \%$ (95\% CI, $0 \%$ to $24 \%$ ) for patients having a first RFI ranging between 1 and 12 months, 23\% (95\% CI, $10 \%$ to 35\%) when the RFI was between 13 and 24 months, 50\% (95\% CI, 32\% to 68\%) when it was between 25 and 36 months, and 74\% (95\% CI, 55\% to $92 \%)$ for patients with a longer RFI $(P<.0001)$. Of the patients with metastases confined to the lung, those with monolateral lung metastases had a slightly better, but not statistically significant, probability of PRS than patients with bilateral lung metastases. A different probability of PRS was observed with regard to the number of resected metastatic nodules: Patients with one to two metastatic nodules had a significantly higher probability of PRS than patients with three or more pulmonary nodules (Table 3).

In the whole group of patients who had complete surgical remission, the use of a second-line chemotherapy treatment did 
Table 3. Probability of Postrelapse Survival and $95 \%$ Confidence Intervals

in 114 Patients With Complete Surgical Remission

\begin{tabular}{|c|c|c|c|c|c|}
\hline \multirow[b]{2}{*}{ Variable } & \multirow{2}{*}{$\begin{array}{l}\text { No. of } \\
\text { Patients }\end{array}$} & \multirow[b]{2}{*}{$\%$ of Survival } & \multicolumn{2}{|c|}{ 5-Year PRS } & \multirow[b]{2}{*}{$P$} \\
\hline & & & $\%$ & $95 \% \mathrm{Cl}$ & \\
\hline Site & & & & & .06 \\
\hline Lung only & 93 & 44 & 44 & 33 to 54 & \\
\hline Other site \pm lung & 21 & 19 & 19 & 1 to 37 & \\
\hline Relapse-free interval & & & & & $<.0001$ \\
\hline$\leq 24$ months & 56 & 18 & 20 & 9 to 30 & \\
\hline$>24$ months & 58 & 60 & 60 & 46 to 74 & \\
\hline Laterality* & & & & & .2 \\
\hline Monolateral & 72 & 46 & 45 & 33 to 57 & \\
\hline Bilateral & 23 & 35 & 37 & 18 to 57 & \\
\hline Lung metastases* & & & & & $<.0001$ \\
\hline$\leq 2$ nodules & 66 & 58 & 59 & 46 to 72 & \\
\hline$>2$ nodules & 29 & 10 & 14 & 1 to 26 & \\
\hline
\end{tabular}

Abbreviation: PRS, postrelapse survival.

*Of 95 patients, 93 had only lung metastases, and two had monolateral lung metastases and local recurrence.

not influence the PRS. A subgroup analysis of PRS according to pattern of recurrence and use of second-line chemotherapy was performed. In the 93 patients with lung metastases only (Table 4), a better PRS for those receiving chemotherapy was observed only in the subgroup of patients with more than two pulmonary nodules; three $(17.5 \%)$ of the 17 patients who received chemotherapy were alive compared with none of the 12 who did not receive chemotherapy (5-year PRS, $18 \%$ and 7\%, respectively; $P=.9$ ). Of the 21 patients with a location of recurrence other than the lung, eight did not have chemotherapy after their relapse, and none survived after 5 years from recurrence. Five of the 13 patients treated with chemotherapy were alive, with a 5-year PRS of $33 \%$.

Multivariate analysis, restricted to patients with lung metastases who had complete surgical remission, was carried out to investigate the prognostic significance of the following variables: RFI ( $\leq 24$ months $v>24$ months), number of resected

Table 4. Postrelapse Survival and Use of Second-Line Chemotherapy According to Pattern of Relapse in 93 Patients With Only Lung Metastases Who Reached Surgical Complete Remission

\begin{tabular}{|c|c|c|c|c|}
\hline \multirow{2}{*}{$\begin{array}{c}\text { Variable/Yes-No } \\
\text { Chemotherapy }\end{array}$} & \multirow{2}{*}{$\begin{array}{l}\text { No. of } \\
\text { Patients }\end{array}$} & \multicolumn{2}{|c|}{ 5-Year PRS } & \multirow[b]{2}{*}{$P$} \\
\hline & & $\%$ & $95 \% \mathrm{Cl}$ & \\
\hline \multicolumn{5}{|l|}{ Monolateral } \\
\hline Yes & 19 & 34 & 7 to 61 & .2 \\
\hline No & 51 & 48 & 34 to 62 & \\
\hline \multicolumn{5}{|l|}{ Bilateral } \\
\hline Yes & 10 & 30 & 0 to 45 & .3 \\
\hline No & 13 & 42 & 24 to 76 & \\
\hline \multicolumn{5}{|l|}{$\leq 2$ nodules } \\
\hline Yes & 12 & 30 & 0 to 61 & .02 \\
\hline No & 52 & 65 & 51 to 78 & \\
\hline \multicolumn{5}{|l|}{$>2$ nodules } \\
\hline Yes & 17 & 18 & 0 to 36 & .9 \\
\hline No & 12 & 7 & 0 to 21 & \\
\hline \multicolumn{5}{|l|}{$\mathrm{RFI} \leq 24$ months } \\
\hline Yes & 16 & 6 & 0 to 18 & .001 \\
\hline No & 28 & 30 & 13 to 48 & \\
\hline \multicolumn{5}{|l|}{$\mathrm{RFI}>24$ months } \\
\hline Yes & 13 & 49 & 19 to 78 & .2 \\
\hline No & 36 & 67 & 51 to 83 & \\
\hline
\end{tabular}

Abbreviations: PRS, postrelapse survival; RFI, relapse-free interval. pulmonary nodules (one or two $v$ three or more), and site of pulmonary nodules (monolateral $v$ bilateral). Predictive factors of PRS were the RFI ( $<24$ months; relative risk, 2.61 [95\% CI, 1.4 to 4.7 ]; $P<.002$ ) and the number of pulmonary nodules (one to two nodules; relative risk, 0.3 [95\% CI, 0.2 to 0.5 ]; $P<$ .0001 ), whereas no statistical significance was reported for the site of pulmonary nodules.

On the basis of these results, we calculated the probability of survival in the population of patients who had lung metastases only and who were in complete surgical remission by combining the risk factors, RFI, and number of lung nodules (Fig 1). Patients with an RFI longer than 24 months and only one to two nodules had a good PRS (5-year PRS, 72\% [95\% CI, 58\% to $86 \%])$. Patients with short RFI ( $<24$ months $)$ and three or more lung nodules had a poor PRS (5-year PRS, 5\% [95\% CI, 0\% to $15 \%])$. An intermediate probability of survival was seen for patients with one to two nodules and a short RFI (5-year PRS, $41 \%$ [ $95 \%$ CI, $20 \%$ to $61 \%$ ]) and for those with a long relapse

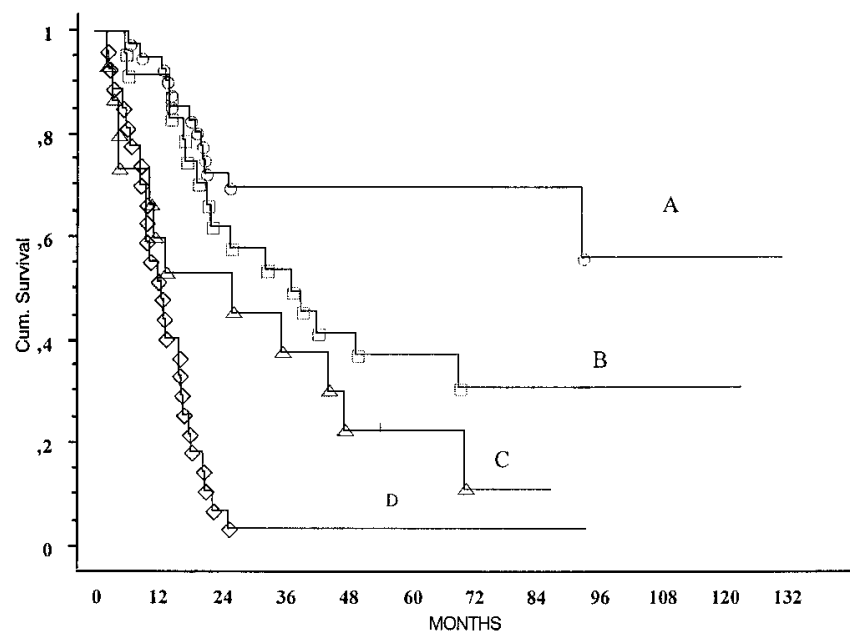

Fig 1. Postrelapse survival by relapse-free interval (RFI) and number of pulmonary metastases (PM) in patients with complete surgery; $A=42$ patients, RFI more than 24 months, 1 to 2 PM; B = 22 patients, RFI less than 24 months; 1 to $2 \mathrm{PM} ; \mathrm{C}=9$ patients, RFI more than 24 months; $\geq 3 \mathrm{PM}$; and D $=20$ patients, RFI less than 24 months; $\geq 3$ PM. 
RFI but three or more nodules (5-year PRS, 33\% [95\% CI, 2\% to $64 \%]$ ).

\section{DISCUSSION}

This study is a retrospective analysis performed on the largest series reported in the literature of patients with extremity osteosarcoma relapsing after complete surgery and neoadjuvant chemotherapy based on the most active drugs against osteosarcoma-high-dose MTX, ADM, CDP, and IFO.

The pattern of recurrence observed in our study was similar to that reported in previous studies ${ }^{11,14,15,17}$ and confirmed that the lungs are the main target of metastases from osteosarcoma. A close relation among pattern of recurrence, surgical resectability of the recurrence, and PRS was observed. In total, 125 patients (77\%) underwent surgery, which resulted in complete resection of the metastatic lesions in 114 of the patients. Therefore, 70\% of the patients had complete surgical remission. The percentage of patients who could be made surgically disease-free was $74.5 \%$ when metastases were confined to the lung compared with 57\% in patients with metastases that were in other locations $(P=$ .044). All 48 patients who did not have complete surgical remission died of disease, whereas those who could be made surgically disease free had a 39\% 5-year probability of survival. In a large series reported by Huth and Eilber, ${ }^{11}$ complete surgery was achieved in $60 \%$ of the patients with metastases confined to the lung and in $31 \%$ of those with metastases in locations other than the lung. The 3-year survival in patients who had complete surgery for lung metastases was $36 \%$, and it was $0 \%$ for those who did not. In the Scandinavian Sarcoma Group experience, ${ }^{15}$ $28(47 \%)$ of 60 metastatic patients underwent complete surgery: $27(51 \%)$ of the 53 patients with lung metastases and one (14\%) of seven patients with recurrence in another site. The 5-year survival for patients who were treated with or without complete surgery was $50 \%$ and $0 \%$, respectively.

The analysis of survival restricted to the patients who achieved a complete surgical remission reinforces the prognostic significance of the pattern of recurrence; the 5-year survival probability was $44 \%$ in case of lung metastases alone and $19 \%$ when recurrence involved other sites. The different pattern of relapse could be the expression of a different biologic behavior of the tumor, indicating that the tumor is more aggressive in patients with tumor recurrence in sites other than the lung, as already reported in the case of local recurrence. ${ }^{21,22}$

Another factor that influenced survival was the RFI. The median RFI observed in our series was 23.5 months, with $5 \%$ of the patients having an RFI longer than 60 months; the latest recurrence occurred after 11 years and 5 months of follow-up. These data show the need for prolonged follow-up in patients with nonmetastatic osteosarcoma. A close relation was found in our series between duration of RFI and PRS; the 5-year PRS was only $8 \%$ in patients with an RFI of 1 year or shorter, and it increased to $23 \%, 50 \%$, and $74 \%$ with a 2 -year RFI, a 3-year RFI, or a longer RFI, respectively. Contrasting data are reported with regard to this issue. In the analysis by Saeter et al, ${ }^{15}$ the RFI, with a cutoff set at 21 months, lost significance after multivariate analysis. Other articles have reported a possible influence of the length of RFI on survival, but only when the cutoff was set at 8 months,${ }^{14}$ whereas in the analysis of Skinner et al ${ }^{13}$ the RFI did not correlate with PRS. In a large analysis on long-term results after resection of lung metastases from different types of malignancies, the RFI significantly influenced the survival, with a cutoff set at 36 months. ${ }^{23}$

The lung was the most common site of osteosarcoma metastases, and in our series, 93 patients with lung metastases underwent complete surgery, which gave them a probability of a 3 - and 5-year survival of 52\% and 44\%, respectively. In a similar population, Huth and Eilber ${ }^{11}$ reported a 3-year PRS of 36\%, Beron et $\mathrm{al}^{9}$ reported a 3-year PRS of 30\%, Skinner et $\mathrm{al}^{13}$ and Belli et al ${ }^{12}$ reported a 5 -year PRS of $37 \%$, and Ward et al ${ }^{14}$ reported a 5-year PRS of $23 \%$. A higher percentage of PRS (5-year PRS, 50\%) was reported by Saeter et al. ${ }^{15}$ However, we must remember that in the Scandinavian Sarcoma Group (SSG) report, only $51 \%$ of the patients with lung metastases underwent complete resection, and a possible selection of patients with a better prognosis might be supposed in the Saeter et al analysis of this subset of patients. In a more recent article,${ }^{16}$ a 3 -year PRS of $61 \%$ was reported.

The number of resected pulmonary nodules was a prognostic factor of PRS, with a significantly better prognosis for patients with one or two lung metastases. Similar results were reported by Saeter et al ${ }^{15}$ Ward et al ${ }^{14}$ and Skinner et al,${ }^{13}$ whereas in the report by Goorin et al, ${ }^{17}$ the number of lung metastases did not influence the PRS.

Subgroups with significantly different prognoses were identified by combining the number of lung metastases resected and the length of RFI. A very good prognosis (5-year PRS, 72\%) was found in patients with RFI longer than 24 months and one or two pulmonary nodules, whereas a very poor prognosis (5-year PRS, $5 \%$ ) was observed in patients with a shorter RFI and three or more metastatic nodules. Patients with one to two pulmonary nodules and short RFI and those with a long RFI but three or more nodules had an intermediate probability of PRS (5-year PRS, $41 \%$ and $33 \%$, respectively). What we observed in patients with a long RFI and one to two pulmonary nodules confirms what was recently reported by Daw et al. ${ }^{24}$

Nowadays, despite the fact that it is quite common to give second-line chemotherapy treatment, the role of this treatment in the management of relapsing osteosarcoma patients is not defined at all, and no controlled studies are available on this topic. However, the rarity of the tumor and the heterogeneous pattern of relapse make it almost impossible to carry out randomized studies. Saeter et $\mathrm{al}^{15}$ reported that adequate chemotherapy (based on drugs not used in first-line therapy) significantly influenced the PRS, whereas Belli et al ${ }^{12}$ failed to find any advantage for patients who received chemotherapy. The results reported in selected patients who underwent complete metastasectomy and received second-line chemotherapy treatment ${ }^{10,14}$ were quite similar to those reported in comparable patients who did not receive chemotherapy. ${ }^{13}$

Some indications of the role of chemotherapy in relapsing osteosarcoma come from our study. It is interesting to notice that unresectable patients who were treated with chemotherapy had a PRS time significantly longer than that observed in patients who refused chemotherapy; however, this was the only subgroup in which second-line chemotherapy gave a significant advantage. In patients who were made surgically disease-free, the addition of 
second-line chemotherapy did not offer any statistically significant advantage in terms of PRS. A possible positive role of chemotherapy could be supposed only for the group of patients with three or more pulmonary nodules (three of 17 patients who received chemotherapy were alive compared with zero of 12 who did not receive chemotherapy). These data are quite disappointing if we consider that more than $50 \%$ of patients received aggressive chemotherapy treatment based on high-dose IFO, the activity of which, at least in terms of response, has been well documented in relapsing sarcoma patients, ${ }^{25}$ and the remaining patients received drugs different from those used in first-line treatment. However, these data come from a retrospective analysis, and the fact that the strategy of treatment was individualized resulted in a selection bias among those who received chemotherapy after tumor resection. Chemotherapy was, in fact, more frequently used in patients whose relapse pattern indicated an aggressive behavior of the tumor.

This retrospective analysis showed that RFI, site of metastases, and number of pulmonary nodules are the main prognostic factors for PRS in osteosarcoma. Complete surgery of recurrence is pivotal in the strategy of treatment. Our data do not support a generalized use of second-line chemotherapy in relapsing osteosarcoma but do support a generalized use only in selected subgroups, such as unresectable patients, and, possibly, those with three or more pulmonary nodules. It is important to stimulate multicenter studies to determine the role of chemotherapy, and at the same time, it is important to develop the research of new drugs that are active against osteosarcoma.

\section{REFERENCES}

1. Campanacci M: Bone and Soft Tissue Tumors. New York, NY, Springer-Verlag, 1999, pp 463-557

2. Bramwell VHC, Burgers M, Sneath R, et al: A comparison of two short intensive adjuvant chemotherapy regimens in operable osteosarcoma of limbs in children and young adults: The first study of the European Osteosarcoma Intergroup. J Clin Oncol 10:1579-1591, 1992

3. Bacci G, Picci P, Ferrari S, et al: Primary chemotherapy and delayed surgery for non metastatic osteosarcoma of the extremity: Results in 164 patients preoperatively treated with high doses of methotrexate, followed by cisplatin and doxorubicin. Cancer 72:1216-1226, 1993

4. Provisor AJ, Ettiger LJ, Nachman JB, et al: Treatment of nonmetastatic osteosarcoma of the extremity with preoperative and postoperative chemotherapy: A report from the Children's Cancer Group. J Clin Oncol 15:76-84, 1997

5. Meyers PA, Gorlick R, Heller G, et al: Intensification of preoperative chemotherapy for osteogenic sarcoma: Results of the Memorial SloanKettering (T-12) protocol. J Clin Oncol 16:2452-2458, 1998

6. Fuchs N, Bielack S, Epler D, et al: Long-term results of the cooperative German-Austrian-Swiss Osteosarcoma Study Group's protocol COSS-86 of intensive multidrug chemotherapy and surgery for osteosarcoma of the limbs. Ann Oncol 9:893-899, 1998

7. Putnam JB, Roth JA, Wesley MN, et al: Survival following aggressive resection of pulmonary metastases from osteogenic sarcoma: Analysis of prognostic factors. Ann Thorac Surg 36:516-523, 1983

8. Goorin AM, Delorey MJ, Lack EE, et al: Prognostic significance of complete surgical resection of pulmonary metastases in patients with osteogenic sarcoma: Analysis of 32 patients. J Clin Oncol 2:425-431, 1984

9. Beron G, Euler A, Winkler K: Pulmonary metastases from osteogenic sarcoma: Complete resection and effective chemotherapy contributing to improved prognosis. Eur Paediatr Haematol Oncol 2:77-85, 1985

10. Meyer WH, Schell MJ, Kumar AP, et al: Thoracotomy for pulmonary metastatic osteosarcoma: An analysis of prognostic indicators of survival. Cancer 59:374-379, 1987

11. Huth JF, Eilber FR: Patterns of recurrence after resection of osteosarcoma of the extremity: Strategies for treatment of metastases. Arch Surg 124:122-126, 1989

12. Belli L, Scholl S, Livartowski A, et al: Resection of pulmonary metastases in osteosarcoma: A retrospective analysis of 44 patients. Cancer 63:2546-2550, 1989

13. Skinner KA, Eilber FR, Holmes EC, et al: Surgical treatment and chemotherapy for pulmonary metastases from osteosarcoma. Arch Surg 127:1065-1071, 1992
14. Ward WG, Mikaelian K, Dorey F, et al: Pulmonary metastases of stage IIB extremity osteosarcoma and subsequent pulmonary metastases. J Clin Oncol 12:1849-1858, 1994

15. Saeter G, Hoie J, Stenwig AE, et al: Systemic relapse of patients with osteogenic sarcoma: Prognostic factors for long term survival. Cancer 75:1084-1093, 1995

16. Antunes M, Bernardo J, Salete M, et al: Excision of pulmonary metastases of osteogenic sarcoma of limbs. Eur J Cardiothorac Surg 15:592-598, 1999

17. Goorin AM, Shuster JJ, Baker A, et al: Changing pattern of pulmonary metastases with adjuvant chemotherapy in patients with osteosarcoma: Results from the multi-institutional osteosarcoma study. J Clin Oncol 9:600-605, 1991

18. Ferrari S, Mercuri M, Picci P, et al: Results of a neoadjuvant chemotherapy protocol (IOR/OS-3) with high-dose methotrexate, intraarterial or intravenous cisplatin, doxorubicin, and salvage chemotherapy based on histologic tumor response. Tumori 85:458-464, 1999

19. Bacci G, Ferrari S, Mercuri M, et al: Neoadjuvant chemotherapy for extremity osteosarcoma: Preliminary results of the Rizzoli's 4th study. Acta Oncol 37:41-48, 1998

20. Bacci G, Ferrari S, Longhi A, et al: Neoadjuvant chemotherapy for high grade osteosarcoma of the extremities: Long-term results for patients treated according to the Rizzoli IOR/OS-3b protocol. J Chemother 13:93-99, 2001

21. Bacci G, Ferrari S, Mercuri M, et al: Predictive factors for local recurrence in osteosarcoma. Acta Orthop Scand 69:230-236, 1998

22. Weeden S, Grimer RJ, Cannon SR, et al: The effect of local recurrence on survival in resected osteosarcoma. Eur J Cancer 37:39-46, 2001

23. Pastorino U, Buyse M, Friedel G, et al: Long-term results of lung metastasectomy: Prognostic analyses based on 5206 cases. J Thorac Cardiovasc Surg 113:37-49, 1997

24. Daw N, Rodriguez-Galindo C, Rao B, et al: Outcome of patients presenting with a single pulmonary metastasis more than one year after diagnosis of osteosarcoma. Proc Am Soc Clin Oncol 19:596, 2000 (abstr 2345)

25. Patel SR, Vadhan-Raj S, Papadopulos N, et al: High-dose ifosfamide in bone and soft tissue sarcomas: Results of phase II and pilot studies-dose response and schedule dependence. J Clin Oncol 15:2378-2384, 1997 